\title{
A novel topical association with zinc oxide, chamomile and aloe vera extracts - stability and safety studies
}

\author{
Uma nova associação de uso tópico contendo óxido de zinco e extractos de aloe vera e \\ camomila - estudos de estabilidade e segurança
}

\section{Catarina Reis ${ }^{1}$, Ana F. Antunes ${ }^{1}$, Patrícia Rijo ${ }^{1}$, Marina Baptista ${ }^{1}$, Joana P Mota ${ }^{1}$, Luís Monteiro Rodrigues ${ }^{1,2}$}

${ }^{1}$ CBIOS (Research Center for Biosciences and Health Technologies), Universidade Lusófona de Humanidade e Tecnologias, Lisboa, Portugal

${ }^{2}$ Pharmacol. Sc Depart - Universidade de Lisboa, School of Pharmacy, Lisboa, Portugal

E-mail: catarina.reis@ulusofona.pt

\begin{abstract}
Currently, natural products show an enormous potential for pharmaceutical and cosmetic industries. The goals of this study were to formulate and to characterise a novel combination of natural products. Formulations were 1) an oil-in-water emulsion, 2) a water-in-oil emulsion and 3) a cleansing solution. Zinc oxide was chosen as an active ingredient due to its healing properties, and chamomile and aloe vera extracts were chosen due to their antiseptic, anti-inflammatory and tissue regenerating properties. Organoleptic characteristics, $\mathrm{pH}$, viscosity and in vitro efficacy for the most common bacteria and yeast of human skin were evaluated. Preliminary and accelerated stability studies and safety tests were also performed. All optimized products were stable, smooth in texture, effective against bacteria and yeast, and safe, justifying further studies. Results suggest that these novel products might be a promising source of natural compounds with soothing and regenerative properties for skin care.
\end{abstract}

Keywords: Chamomilla recutita; Aloe vera; Zinc Oxide; Topical formulations; Skin.

\section{Resumo:}

Atualmente, os produtos naturais apresentam um enorme potencial para as indústrias farmacêuticas e cosméticas. Os objetivos deste estudo foram a formulação e a caracterização de três cosméticos contendo produtos naturais. As formulações foram: 1) emulsão óleo-em-água; 2) emulsão água-em-óleo e 3) solução de limpeza. O óxido de zinco foi escolhido como ingrediente ativo devido às suas propriedades regenerativas e os extratos de aloe vera e camomila devido às suas propriedades antissépticas, anti-inflamatórias e também regenerativas. Os parâmetros avaliados foram as características organoléticas, $\mathrm{pH}$, viscosidade e a eficácia in vitro contra bactérias e fungos comuns em doenças de pele. Foram igualmente efetuados estudos preliminares e acelerados de estabilidade e segurança. Todos os produtos demonstraram estabilidade, textura suave, eficácia contra bactérias e fungos e segurança, justificando estudos adicionais. Os resultados obtidos sugerem que estes novos produtos poderão ser uma fonte promissora de produtos naturais com propriedades calmante e regenerativa para a pele.

Palavras-chave: Chamomilla recutita; Aloe vera; Óxido de zinco; Formulações tópicas; Pele. 


\section{Introduction}

Ointment bases may have a great importance in terms of skin protection and permeation since it is clear that these topical formulations are actually a major determinant of skin care and dermatological health, also favoring aesthetical appearance, hence retarding skin ageing ${ }^{(1,2)}$. In general, these bases can be set in five different types with distinct physical properties. Specifically, bases for transdermal drug delivery can be classified in hydrophobic, absorbent, water-soluble and water-in-oil $(\mathrm{W} / \mathrm{O})$ or oil-in-water $(\mathrm{O} / \mathrm{W})$ semi-solid formulations, with the oil-in-water formulations often used for preparing creams. In this study, we developed three different formulations $(\mathrm{O} / \mathrm{W}$ emulsion, $\mathrm{W} / \mathrm{O}$ emulsion and cleansing solution) with an original combination of natural products known for their soothing, regenerating, anti-inflammatory and/or antiseptic properties ${ }^{(3-6)}$. All products were further characterised for organoleptic, $\mathrm{pH}$, viscosity and in vitro efficacy against the most frequent bacteria and yeast in human skin diseases. Preliminary stability studies and safety assessment were also performed.

\section{Materials and Methods}

All reagents were analytical grade.

\section{a) Production of $W / O$ emulsion}

A W/O emulsion was prepared by weighing the constituents of the oily phase containing vitamin $\mathrm{E}$ acetate $(2.94-3.2 \%$, w/v), dimethicone $(0.98-1.1 \%$, w/v), Dra$\operatorname{gosan}{ }^{\circledR}(1.96-6.00 \%, \mathrm{w} / \mathrm{v})$, lanolin $(5.3-10.0 \%, \mathrm{w} / \mathrm{v})$, cod liver oil $(2.7-12.55 \%, \mathrm{w} / \mathrm{v})$ and white petroleum $(19.6-21.4 \%, \mathrm{w} / \mathrm{v})^{(7-9)}$. These components were melted in a water bath at $65{ }^{\circ} \mathrm{C}$ and slowly mixed. The aqueous phase included citric acid $(0.49-0.52 \%, \mathrm{w} / \mathrm{v})$, water $(21$ $-23 \%, \mathrm{w} / \mathrm{v})$, magnesium sulfate $(0.98-1.1 \%, \mathrm{w} / \mathrm{v})$, Neolon $950 \AA(0.736 \%, \mathrm{w} / \mathrm{v})$ and $\mathrm{Abiol} \AA(0.3 \%, \mathrm{w} / \mathrm{v})$, which were accurately weighed and heated in water bath at 65 ${ }^{\circ} \mathrm{C}{ }^{(1,7,10)}$. Afterward, the previous two phases were mixed for 20 minutes until a homogenous emulsion was formed. After cooling the temperature to $60^{\circ} \mathrm{C}$, chamomile $(0.49$ $0.52 \%, \mathrm{w} / \mathrm{v})$ and aloe vera extracts $(4.9-5.3 \%, \mathrm{w} / \mathrm{v})$ were added ${ }^{(4-6,11,12)}$. In a pre-heated $\left(60^{\circ} \mathrm{C}\right)$ mortar, corn starch $(14.7-16.0 \%, \mathrm{w} / \mathrm{v})$, titanium oxide $(1.5-1.6 \%, \mathrm{w} / \mathrm{v})$ and zinc oxide $\left(14.7-16.0 \%\right.$, w/v) were added ${ }^{(7,9,13)}$, after which the $\mathrm{W} / \mathrm{O}$ emulsion was homogenized and packaged. Thirteen formulations were prepared according to Table 1.

\section{Introdução}

Os produtos cosméticos têm uma grande importância em termos de protecção da pele e permeação cutânea uma vez que é claro que as formulações tópicas são, na verdade, um dos principais determinantes de cuidados da pele e saúde dermatológica, favorecendo a aparência estética e, portanto, poderão retardar o envelhecimento da pele ${ }^{(1,2)}$. Geralmente, as bases podem ser de cinco tipos diferentes com propriedades físicas distintas. Mais especificamente, as bases utilizadas para veiculação transdérmica de fármacos podem ser classificadas em hidrofóbicas, absorventes, hidrossolúveis ou formulações semi-sólidas água em óleo $(\mathrm{A} / \mathrm{O})$ ou óleo em água $(\mathrm{O} / \mathrm{A})$, sendo esta última frequentemente usada na preparação de cremes. Neste estudo, foram desenvolvidos três produtos cosméticos diferentes (emulsão $\mathrm{O} / \mathrm{A}$, emulsão $\mathrm{A} / \mathrm{O}$ e solução de limpeza) que têm na sua composição produtos naturais com propriedades calmante, anti-inflamatória, antisséptica e de regeneração de tecidos ${ }^{(3-6)}$. Todos os produtos foram caracterizados em termos de propriedades organoléticas, $\mathrm{pH}$, viscosidade e eficácia in vitro contra as bactérias e fungos mais frequentemente associadas com doenças da pele. Foram igualmente efetuados estudos preliminares e acelerados de estabilidade e segurança com voluntários humanos.

\section{Materiais e Métodos}

Todos os reagentes utilizados foram de grau analítico.

\section{a) Produção da emulsão $A / O$}

A emulsão $\mathrm{A} / \mathrm{O}$ foi preparada através da pesagem dos constituintes da fase oleosa composta por vitamina $\mathrm{E}$ $(2,94-3,2 \%, \mathrm{p} / \mathrm{v})$, dimeticona $(0,98-1,1 \%, \mathrm{p} / \mathrm{v})$, Dragosan® $(1,96-6,0 \%, p / v)$, lanolina $(5,3-10,0 \%, p / v)$, óleo de fígado de bacalhau $(2,7-12,55 \%, \mathrm{p} / \mathrm{v})$ e vaselina branca $(19,6-21,4 \%, p / v)^{(7-9)}$. Estes componentes foram dissolvidos num banho de água a $65^{\circ} \mathrm{C}$ e misturados lentamente. Os componentes da fase aquosa, incluindo o ácido cítrico $(0,49-0,52 \%, \mathrm{p} / \mathrm{v})$, água $(21-23 \%, \mathrm{p} / \mathrm{v})$, sulfato de magnésio $(0,98-1,1 \%, \mathrm{p} / \mathrm{v})$, Neolon $950 \AA(0,736 \%, \mathrm{p} / \mathrm{v})$ e Abiol ${ }^{\circledR}(0,3 \%, p / v)$, foram pesados e aquecidos num banho de água a $65^{\circ} \mathrm{C}^{(1,7,10)}$. Posteriormente, misturaram-se as duas fases durante 20 minutos até formação de uma emulsão homogénea. Após arrefecimento até $60^{\circ} \mathrm{C}$, adicionaram-se extratos de camomila $(0,49-0,52 \%, \mathrm{p} / \mathrm{v}) \mathrm{e}$ de aloé vera $(4,9-5,3 \%, \mathrm{p} / \mathrm{v})^{(4-6,11-12)}$. Num almofariz pré-aquecido $\left(60{ }^{\circ} \mathrm{C}\right)$, adicionou-se amido de milho $(14,7-$ $16,0 \%, \mathrm{p} / \mathrm{v})$, óxido de titânio $(1,5-1,6 \%, \mathrm{p} / \mathrm{v})$ e óxido de zinco $(14,7-16,0 \%, \mathrm{p} / \mathrm{v})^{(7,9,13)}$. Por fim, as emulsões $\mathrm{A} / \mathrm{O}$ foram homogeneizadas e embaladas. Foram preparadas 13 formulações de acordo com a Tabela 1 . 
Table 1/ Tabela 1. Composition of the W/O emulsions Composição da emulsão A/O.

\begin{tabular}{|c|c|c|c|c|c|c|c|c|c|c|c|c|c|}
\hline \multirow{2}{*}{$\begin{array}{l}\text { Ingredients/ } \\
\text { Ingredientes }\end{array}$} & \multicolumn{13}{|c|}{ Formulations/Formulações } \\
\hline & I & II & III & IV & $\mathbf{V}$ & VI & VII & VIII & IX & $\mathbf{X}$ & $\mathbf{X I}$ & XII & XIII \\
\hline $\begin{array}{c}\text { Zinc Oxide/ } \\
\text { Óxido de Zinco }\end{array}$ & 15 & & & - & & & + & & - & + & & & + \\
\hline $\begin{array}{c}\text { White } \\
\text { Petroleum/ } \\
\text { Vaselina Branca }\end{array}$ & 30.1 & - & & - & & & + & & - & + & & & - \\
\hline $\begin{array}{l}\text { Lanolin/ } \\
\text { Lanolina }\end{array}$ & 10 & - & & - & & & + & & - & - & & & - \\
\hline $\begin{array}{c}\text { Chamomilla } \\
\text { extract/ } \\
\text { Extrato de } \\
\text { camomila }\end{array}$ & 0.5 & & & - & & & + & & - & & & & \\
\hline $\begin{array}{c}\text { Aloe vera } \\
\text { extract/ } \\
\text { Extracto Aloe } \\
\text { vera } \\
\end{array}$ & 5 & & & - & & & + & & - & + & & & + \\
\hline $\begin{array}{c}\text { Vitamin E } \\
\text { Acetate/ } \\
\text { Acetato de } \\
\text { Vitamina E }\end{array}$ & 3 & & & - & & & + & & & + & & & + \\
\hline $\begin{array}{c}\text { Cod liver oil/ } \\
\text { Óleo fígado de } \\
\text { bacalhau }\end{array}$ & 12.55 & - & & - & & & + & & - & - & & & - \\
\hline BHT & 0.1 & & & & & & & & & & & & \\
\hline BHA & 0.02 & & & & & & & & & & & & \\
\hline $\begin{array}{c}\text { Corn Starch/ } \\
\text { Amido de milho }\end{array}$ & 15 & & & + & - & & + & & - & + & & & + \\
\hline $\begin{array}{c}\text { Dimethicone } \\
\text { Dimeticona }\end{array}$ & 1 & & & - & & & + & & & + & & & + \\
\hline $\begin{array}{l}\text { Magnesium } \\
\text { sulfate/ } \\
\text { Sulfato de } \\
\text { magnésio }\end{array}$ & 1 & & & - & & & + & & & + & & & + \\
\hline $\begin{array}{l}\text { Citric acid/ } \\
\text { Ácido cítrico }\end{array}$ & 0.5 & & & - & & & + & & - & & & & \\
\hline Dragosan W/O & 6 & - & & - & & & + & & - & + & & & - \\
\hline $\begin{array}{l}\text { Water/ } \\
\text { Água }\end{array}$ & & 21.6 & - & - & & & + & & - & + & & - & + \\
\hline $\begin{array}{l}\text { Titanium Oxide/ } \\
\text { Óxido de titânio }\end{array}$ & & & & & & & & & 1.5 & + & & & + \\
\hline Silica & & & & & 4.9 & & & & & & & & \\
\hline $\begin{array}{l}\text { Talc/ } \\
\text { Talco }\end{array}$ & & & & & & 4.9 & & & & & & & \\
\hline Geogard ultra & & & & & & & & & & & & 1 & \\
\hline Abiol ${ }^{\circledR}$ & & & & & & & & & & & & & 0.3 \\
\hline Neolon $950 \AA$ & & & & & & & & & & & & & 0.736 \\
\hline
\end{tabular}




\section{b) Production of $\mathrm{O} / \mathrm{W}$ emulsion}

An $\mathrm{O} / \mathrm{W}$ emulsion was prepared by weighing the constituents of the oily phase composed of vitamin E acetate $(0.75-3.0 \%, \mathrm{w} / \mathrm{v})$, cetearyl alcohol $(1.9-2.5 \%$, w/v), C12-15 Alkyl benzoate (2.86 - 3.7\%, w/v), caprylic-capric triglyceride $(6.67-8.6 \%, \mathrm{w} / \mathrm{v})$, cetearyl ethylhexanoate $(4.76-6.5 \%, \mathrm{w} / \mathrm{v})$, dexpanthenol $(0.3$ $1.0 \%, \mathrm{w} / \mathrm{v})$, dimethicone $(9.3-16.0 \%, \mathrm{w} / \mathrm{v})$, shea butter $(1.2-6.67 \%, \mathrm{w} / \mathrm{v})$ and Sabowax $(4.76-6.2 \%, \mathrm{w} / \mathrm{v})^{(7,14)}$. All components were then melted in a water bath at 65 ${ }^{\circ} \mathrm{C}$ and slowly mixed, while the aqueous phase ingredients were weighed and heated in a water bath at 65 ${ }^{\circ} \mathrm{C}$. The aqueous phase includes propylene glycol (2.86 - $3.70 \%$, w/v), water $(37.62-44.4 \%$, w/v), Neolon $950 \AA(0.736 \%, \mathrm{w} / \mathrm{v})$ and Abiol ${ }^{\circledR}(0.3 \%, \mathrm{w} / \mathrm{v}){ }^{(7,10)}$. The two phases were then mixed for 20 minutes until the emulsion was homogeneous. After cooling the temperature to $60{ }^{\circ} \mathrm{C}$, chamomile $(0.48-0.6 \%, \mathrm{w} / \mathrm{v})$ and aloe vera extracts $\left(4.76-6.2 \%\right.$, w/v) were added ${ }^{(4-6,11,12)}$. In pre-heated $\left(60^{\circ} \mathrm{C}\right)$ mortar, zinc oxide $(4.76-6.2 \%, \mathrm{w} / \mathrm{v})$ was also added ${ }^{(9,13}$ we have advanced in our development of a novel diaper that delivers dermatological formulations to help protect the skin from over-hydration and irritation. $\mid n \backslash$ nOBJECTIVE: To determine the clinical benefits of a novel disposable diaper designed to deliver a zinc oxide and petrolatum-based formulation continuously to the skin during use. $\backslash n \backslash n$ METHODS: All studies were independent, blinded, randomized clinical trials. Study A was conducted to confirm transfer of the zinc oxide/petrolatum ( $\mathrm{ZnO} /$ Pet). The $\mathrm{W} / \mathrm{O}$ emulsion was then homogenized and packaged. Eleven different were prepared according to Table 2 .

\section{c) Development of a cleansing solution}

The solution was prepared by dissolving sodium chloride $(0.9 \%, \mathrm{w} / \mathrm{v})$ in distilled water which was previously mixed with sodium hypochlorite $(0.01 \%$, w/v). Chamomile $(0.5 \%, \mathrm{w} / \mathrm{v})$ and aloe vera extracts $(5.0 \%, \mathrm{w} / \mathrm{v})$ were then added, followed by $\mathrm{pH}$ adjustment (to $\mathrm{pH}$ 4.7) and clarification by filtration. Finally, $1 \mu \mathrm{L}$ of green dye was added per $\mathrm{mL}$ of solution for a body cleansing solution and $0.4 \mu \mathrm{L}$ of attar of roses per $\mathrm{mL}$ of solution for the intimate cleansing solution.

\section{.Characterization of preparations}

Physical characterization of the developed formulations involved the organoleptic evaluation of color, odour and texture. The best scores, regarded as best formulations, were chosen for additional testing regarding primary stability, accelerated stability, viscosity, $\mathrm{pH}$, in vitro efficacy and safety.

\section{b) Produção da emulsão $O / A$}

A emulsão $\mathrm{O} / \mathrm{A}$ foi preparada através da pesagem dos constituintes da fase oleosa composta por acetato de vitamina $\mathrm{E}(0,75-3,00 \%, \mathrm{p} / \mathrm{v})$, álcool cetoestearílico $(1,9-$ $2,5 \%, \mathrm{p} / \mathrm{v}), \mathrm{C} 12-15$ Benzoato de alquilo $(2,86-3,70 \%$, $\mathrm{p} / \mathrm{v})$, triglicéridos caprílico-cáprico $(6,67-8,60 \%, \mathrm{p} / \mathrm{v})$, etil-hexanoato cetoesterílico $(4,76-6,50 \%, \mathrm{p} / \mathrm{v})$, dexpantenol $(0,3-1 \%, \mathrm{p} / \mathrm{v})$, dimeticona $(9,3-16,0 \%, \mathrm{p} / \mathrm{v})$, manteiga de karité $(1,20-6,67 \%, p / v)$ e Sabowax ${ }^{\circledR}$ $(4,76-6,2 \%, p / v)^{(7,14)}$. Estes componentes foram dissolvidos num banho de água a $65^{\circ} \mathrm{C}$ e misturados lentamente. Os componentes da fase aquosa incluindo, propilenoglicol $(2,86-3,70 \%, \mathrm{w} / \mathrm{v})$, água $(37,62-44,4 \%$, $\mathrm{p} / \mathrm{v})$, Neolon $950 \AA(0,736 \%, \mathrm{p} / \mathrm{v})$ e Abiol ${ }^{\circledR}(0,3 \%$, $\mathrm{p} / \mathrm{v})$, foram pesados e aquecidos num banho de água a $65^{\circ} \mathrm{C}^{(7,10)}$. Posteriormente, misturaram-se as duas fases durante 20 minutos até formação de uma emulsão homogénea. Após arrefecimento até $60^{\circ} \mathrm{C}$, adicionaram-se extratos de camomila $(0,48-0,60 \%, \mathrm{p} / \mathrm{v})$ e de aloé vera $(4,76-6,20 \%, \mathrm{p} / \mathrm{v})^{(4-6,11-12)}$. Num almofariz pré-aquecido $\left(60^{\circ} \mathrm{C}\right)$, adicionou-se ainda óxido de zinco $(4,76-6,20 \%$, $\mathrm{p} / \mathrm{v}){ }^{(9,13}$ we have advanced in our development of a novel diaper that delivers dermatological formulations to help protect the skin from over-hydration and irritation. $\mid n \backslash$ nOBJECTIVE: To determine the clinical benefits of a novel disposable diaper designed to deliver a zinc oxide and petrolatum-based formulation continuously to the skin during use. $\backslash n \backslash n M E T H O D S:$ All studies were independent, blinded, randomized clinical trials. Study A was conducted to confirm transfer of the zinc oxide/ petrolatum $(\mathrm{ZnO} / \mathrm{Pet})$. Por fim, as emulsões $\mathrm{A} / \mathrm{O}$ foram homogeneizadas e embaladas. Foram preparadas 11 formulações diferentes de acordo com a Tabela 2.

\section{c) Desenvolvimento da solução de limpeza}

A solução foi preparada dissolvendo cloreto de sódio $(0,9 \%, \mathrm{p} / \mathrm{v})$ em água destilada, a qual tinha sido previamente misturada com hipoclorito de sódio $(0,01 \%$, $\mathrm{p} / \mathrm{v})$. Após solubilização, foram adicionados extratos de camomila $(0,5 \%, \mathrm{p} / \mathrm{v})$ e de aloé vera $(5 \%, \mathrm{p} / \mathrm{v})$, seguido de ajuste do $\mathrm{pH}(\mathrm{pH} 4,7)$ e clarificação por filtração. Finalmente, foi adicionado $1 \mu \mathrm{L}$ de corante verde por $\mathrm{mL}$ de solução de limpeza corporal ou $0,4 \mu \mathrm{L}$ de água de rosas por $\mathrm{mL}$ de solução de limpeza

\section{.Caracterização das formulações}

A caracterização física das formulações desenvolvidas foi efetuada pela avaliação das propriedades organoléticas, tais como cor, odor e textura. As melhores formulações foram testadas em termos de estabilidade preliminar, estabilidade acelerada, viscosidade, $\mathrm{pH}$, eficácia in vitro e avaliação da segurança. 
Table 2/ Tabela 2. Composition of the $\mathrm{O} / \mathrm{W}$ emulsions

Composição da emulsão O/A.

\begin{tabular}{|c|c|c|c|c|c|c|c|c|c|c|c|}
\hline \multirow{2}{*}{$\begin{array}{c}\text { Ingredients } \\
\text { Ingredientes }\end{array}$} & \multicolumn{11}{|c|}{ Formulations/Formulações } \\
\hline & I & II & III & IV & V & VI & VII & VIII & IX & $\mathbf{X}$ & $\mathbf{X I}$ \\
\hline $\begin{array}{c}\text { Zinc Oxide } \\
\text { Óxido de Zinco }\end{array}$ & 4.76 & + & & & + & & + & + & & & + \\
\hline $\begin{array}{c}\text { Cetearyl } \\
\text { ethylhexanoate } \\
\text { Etil-hexanoato } \\
\text { cetoesterílico }\end{array}$ & 4.76 & + & & & + & & + & + & + & - & + \\
\hline $\begin{array}{c}\text { C12-15 Alkyl } \\
\text { Benzoate } \\
\text { C12-15 Benzoato de } \\
\text { alquilo }\end{array}$ & 2.86 & + & & & + & & + & + & - & + & + \\
\hline $\begin{array}{c}\text { Caprylic-capric } \\
\text { triglyceride } \\
\text { Triglicérido caprílico- } \\
\text { cáprico }\end{array}$ & 6.67 & + & & & + & & + & + & & & + \\
\hline $\begin{array}{c}\text { Shea butter } \\
\text { Manteiga de Karité }\end{array}$ & 6.67 & - & - & & - & & & - & + & - & - \\
\hline $\begin{array}{c}\text { Vitamin E Acetate } \\
\text { Acetato de vitamina } \mathrm{E}\end{array}$ & 2.86 & + & & & - & & & - & - & + & - \\
\hline $\begin{array}{l}\text { Chamomilla extract } \\
\text { Extrato de camomila }\end{array}$ & 0.48 & + & & & & & & + & & & + \\
\hline $\begin{array}{c}\text { Aloe vera extract } \\
\text { Extrato de Aloe vera }\end{array}$ & 4.76 & + & & & + & & + & + & & & + \\
\hline Sabowax Sb & 4.76 & + & & & + & & + & + & & & + \\
\hline $\begin{array}{l}\text { Dimethicone } \\
\text { Dimeticona }\end{array}$ & 14.29 & + & + & & + & & + & - & & & + \\
\hline $\begin{array}{l}\text { Cetearyl Alcohol } \\
\text { Álcool cetoestearílico }\end{array}$ & 1.90 & + & & & + & & & + & & & + \\
\hline $\begin{array}{l}\text { Dexpanthenol } \\
\text { Dexpantenol }\end{array}$ & 0.95 & + & & & - & & & - & & & - \\
\hline $\begin{array}{l}\text { Propylene glycol } \\
\text { Propilenoglicol }\end{array}$ & 2.86 & + & & & + & & + & + & & & + \\
\hline $\begin{array}{l}\text { Water } \\
\text { Água }\end{array}$ & 37.62 & + & & & + & & + & + & & - & + \\
\hline $\begin{array}{l}\text { Titanium Oxide } \\
\text { Óxido de Titânio }\end{array}$ & & & 1.5 & & + & & & & & & + \\
\hline Geogard ultra & 1.90 & & & & & & & & & 1 & - \\
\hline Abiol ${ }^{\circledR}$ & & & & & & & & & & & 0.3 \\
\hline Neolon $\left.950^{(}\right)$ & & & & & & & & & & & 0.736 \\
\hline
\end{tabular}




\section{a) Organoleptic characteristics}

All samples were assessed by visual inspection and by microscopy when applicable (color). Odour and appearance were also evaluated.

\section{b) Physicochemical tests}

i. $p H$ : Determination of the $\mathrm{pH}$ was performed at room temperature in triplicate using potentiometer (Metrohm $827 \mathrm{pH}$ lab, Chennai, India).

ii. Viscosity: Viscosity was determined at room temperature using rotational viscometer (Brookfield Viscosimeter, Middeborough, USA) with the $\mathrm{n} .{ }^{\circ} 5$ needle with a speed rate of $1 \mathrm{rpm}$ and with force torque greater than $20 \%$.

\section{c) In vitro efficacy of preservatives}

In vitro efficacy was performed in $\mathrm{O} / \mathrm{W}$ and $\mathrm{W} / \mathrm{O}$ emulsions according with the Portuguese Pharmacopoeia 9.0 ${ }^{(15)}$. Test microorganisms were Pseudomonas aeruginosa (ATCC 9027), Staphylococcus aureus (ATCC 6538), Candida albicans (ATCC 10231) and Aspergillus brasiliensis (ATCC 16404).

\section{d) Preliminary stability}

i. Heating and cooling: Samples were submitted to heat-freeze cycles $\left[25 \pm 2^{\circ} \mathrm{C}\right.$ in an oven (24 hours), then cooled to $-10 \pm 2{ }^{\circ} \mathrm{C}$ in a freezer ( 24 hours)], over seven days. Samples were analyzed at 48, 96 and 168 hours for organoleptic properties.

ii. Centrifuge: The phase separation of all samples by was evaluated by centrifugation. Ten grams of each sample were exposed to a $50^{\circ} \mathrm{C}$ water bath and subsequently centrifuged using a speed rate of $3000 \mathrm{rpm}$ (approximately $1077 \mathrm{x} \mathrm{g}$ ) for 30 minutes.

\section{e) Accelerated stability}

$i$. Tests cycles of heating and cooling: Samples were incubated at $45 \pm 2^{\circ} \mathrm{C}$ in the oven and cooled in the freezer at $-10 \pm 2^{\circ} \mathrm{C}$ (cycles of 24 hours in each condition) for twelve days. Samples were analyzed every two days. This analysis included the evaluation of the organoleptic characteristics.

ii. Stability test during 14 days: Samples (in triplicate) were exposed to three different settings: room temperature $\left(20 \pm 5^{\circ} \mathrm{C}\right)$, refrigerated $\left(-5 \pm 2^{\circ} \mathrm{C}\right)$ and oven $(50 \pm$ $2^{\circ} \mathrm{C}$ ) for 14 days. After 3, 7 and 14 days, formulations were analyzed in terms of organoleptic characteristics, $\mathrm{pH}$ and viscosity.

iii. Test of temperature cycles: Samples were exposed to water bath at $40^{\circ} \mathrm{C}$ with a controlled heating rate of $10^{\circ} \mathrm{C} / 30 \mathrm{~min}$ of up to $80^{\circ} \mathrm{C}$. After returning to room tem-

\section{a) Características organoléticas}

Todas as amostras foram avaliadas por observação visual e, quando necessário, através do microscópio. Também se procedeu à avaliação do odor e aspeto.

\section{b) Testes fisico-químicos}

i) $\mathrm{pH}$ : A determinação do $\mathrm{pH}$ foi efetuada à temperatura ambiente, em triplicado, usando um potenciómetro (Metrohm 827 pH lab, Chennai, Índia).

ii) Viscosidade: A viscosidade foi medida, à temperatura ambiente através de um viscosímetro rotacional (Brookfield Viscosimeter, Middeborough, EUA) com uma agulha n. 5 e a uma velocidade de $1 \mathrm{rpm}$ (rotações por minuto) com força torque superior a $20 \%$.

c) Eficácia in vitro dos conservantes

A eficácia in vitro foi efetuada para as emulsões O/A e A/O de acordo com a Farmacopeia Portuguesa 9.0 ${ }^{(15)}$. Os microrganismos testados foram Pseudomonas aeruginosa (ATCC 9027), Staphylococcus aureus (ATCC 6538), Candida albicans (ATCC 10231) e Aspergillus brasiliensis (ATCC 16404).

\section{d) Estabilidade preliminar das emulsões}

i) Aquecimento e arrefecimento: As amostras foram sujeitas a ciclos de calor e frio durante sete dias $\left(25 \pm 2^{\circ} \mathrm{C}\right.$ numa estufa $(24 \mathrm{~h})$ e arrefecidas até $-10 \pm 2{ }^{\circ} \mathrm{C}$ num congelador $(24 \mathrm{~h})$. As propriedades organoléticas das amostras foram analisadas às 48, 96 e 168 horas.

ii) Centrifugação: A separação de fases foi avaliada por centrifugação. Dez gramas de cada amostra foram expostas a $50^{\circ} \mathrm{C}$ num banho de água e centrifugadas a uma velocidade de $3000 \mathrm{rpm}$ (aproximadamente $1077 \mathrm{x} \mathrm{g}$ ) durante 30 minutos.

\section{e) Estabilidade acelerada das emulsões}

i) Teste de ciclos de aquecimento e arrefecimento: Procedeu-se à incubação das amostras a $45 \pm 2{ }^{\circ} \mathrm{C}$ numa estufa e arrefecimento num congelador a $-10 \pm 2^{\circ} \mathrm{C}$ (ciclos de 24 horas para cada condição) durante 12 dias. As amostras foram analisadas a cada dois dias. Esta análise incluiu a avaliação das características organoléticas.

ii) Teste de estabilidade durante 14 dias: As amostras (em triplicado) foram expostas a três condições: temperatura ambiente $\left(20 \pm 5^{\circ} \mathrm{C}\right)$, refrigeração $\left(-5 \pm 2^{\circ} \mathrm{C}\right) \mathrm{e}$ estufa $\left(50 \pm 2^{\circ} \mathrm{C}\right)$ durante 14 dias. Após 3, 7 e 14 dias, analisaram-se as formulações em termos de características organoléticas, $\mathrm{pH}$ e viscosidade.

iii) Teste de ciclos de temperatura: As amostras foram expostas a $40^{\circ} \mathrm{C}$ em banho de água com uma velocidade de aquecimento controlada de $10^{\circ} \mathrm{C} / 30 \mathrm{~min}$ até $80^{\circ} \mathrm{C}$. 
perature, the samples were analyzed in terms of the $\mathrm{pH}$ and organoleptic characteristics.

\section{f) Preliminary safety test}

The safety of the formulations was assessed by a primary irritation patch test ${ }^{(16,17)}$. Twelve healthy females age between 20 - 40 (mean 24) years old participated in this study after informed written consent. Experiments took place in a room with controlled temperature $(21+$ $2^{\circ} \mathrm{C}$ ) and humidity (40 - 60\%). All procedures complied with the Helsinki declaration and subsequent amendments ${ }^{(18)}$.

\section{Results}

\section{a) Production and characterization}

According to Figure 1, the W/O emulsions had white color, smooth consistency and was homogeneous, without any aggregates. For further analyses, only formulations IX and XIII were further studied. In case of $\mathrm{O} / \mathrm{W}$ emulsions, only V and XI were tested (Figure 2). The cleansing solution proved to be clear and without aggregates as shown in Figure 3.
Após arrefecimento até à temperatura ambiente, analisaram-se as amostras em termos de $\mathrm{pH}$ e características organoléticas.

\section{f) Teste preliminar de segurança}

A segurança foi avaliada através do teste oclusivo (irritação primária da pele) ${ }^{(16,17)}$. Doze mulheres saudáveis com idades compreendidas entre 20-40 (média de 24) anos participaram neste estudo após consentimento informado por escrito. A experiência decorreu em ambiente controlado (temperatura $21+2^{\circ} \mathrm{C}$ e humidade $40-60 \%$ ) e todos os procedimentos foram realizados de acordo com a declaração de Helsínquia e aalterações posteriores ${ }^{(18)}$.

\section{Resultados}

\section{a) Produção e caracterização dos cosméticos}

De acordo com a Figura 1, as formulações A/O apresentavam coloração branca, consistência, suave e homogénea sem agregados. De acordo esta última análise, apenas as formulações IX e XIII prosseguiram os restantes ensaios. No caso das emulsões $\mathrm{A} / \mathrm{O}$, apenas as formulações V e XI foram testadas (Figura 2). Como pode ser observado na Figura 3, a solução de limpeza revelou-se límpida e sem agregados visíveis.
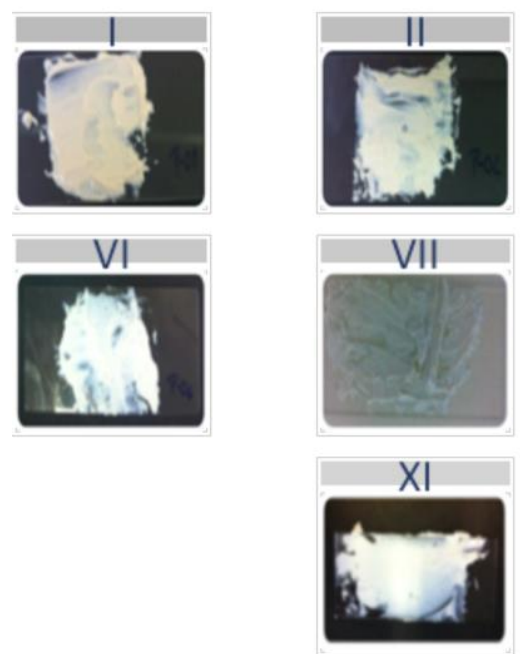
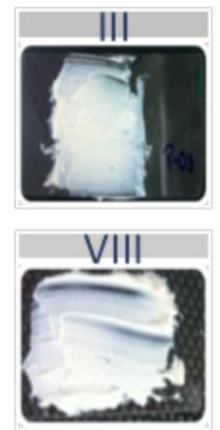

XII

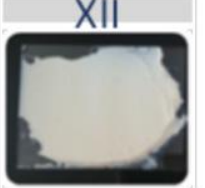

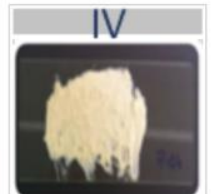
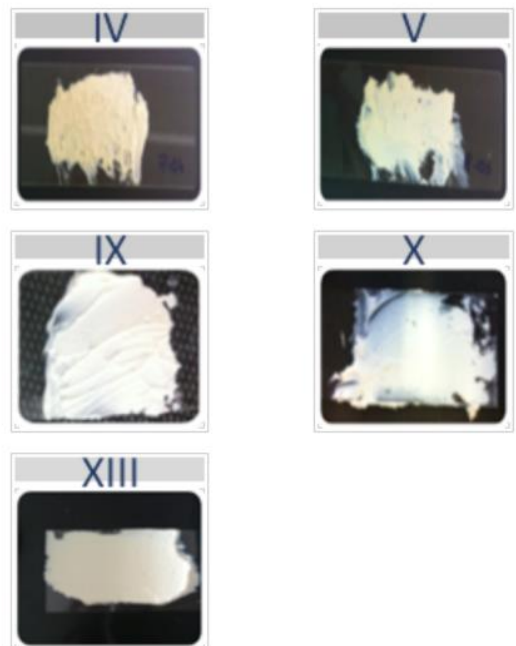

Figure 1/ Figura 1. W/O formulations

Formulações A/O. 

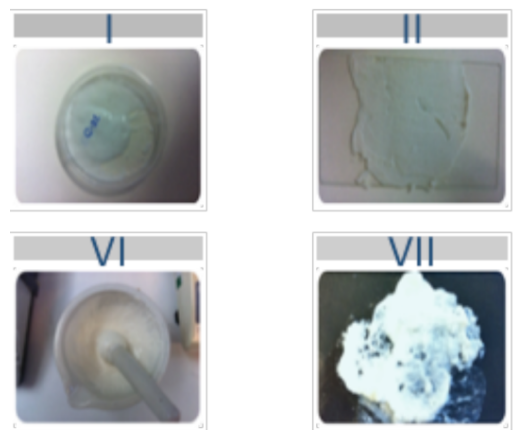
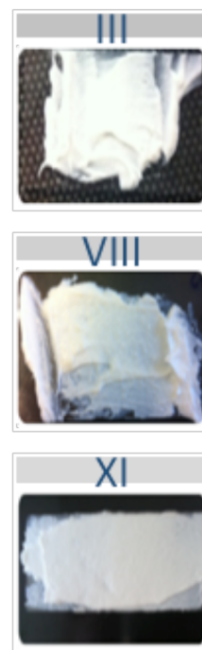

Figure 2/ Figura 2. O/W formulations Formulações $\mathrm{O} / \mathrm{A}$.
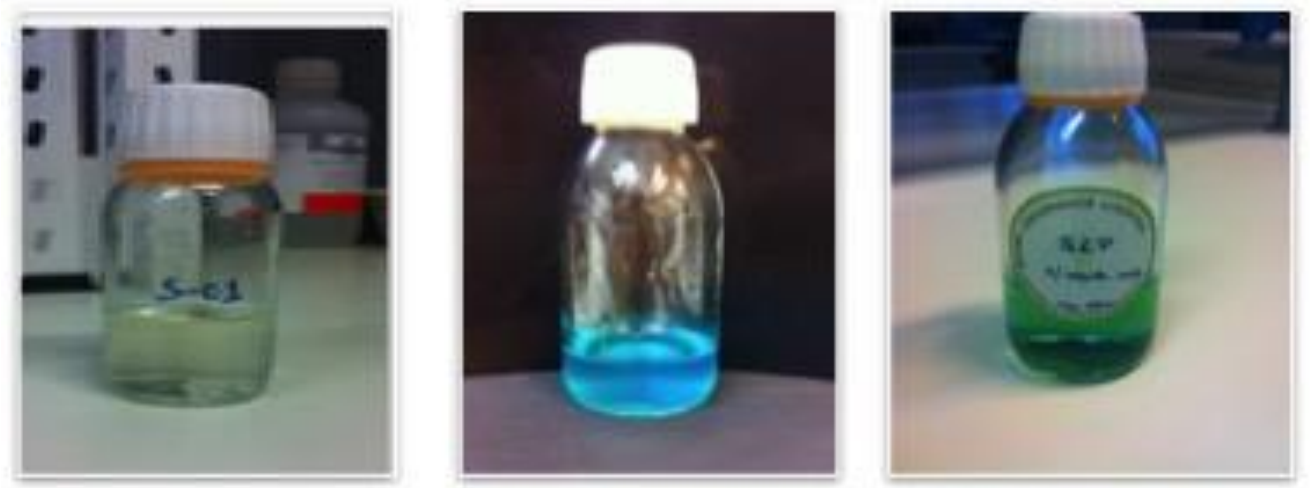

Figure 3/ Figura 3. Cleansing solution without and with () dyes Solução de limpeza sem e com corantes.

b) In vitro efficacy of emulsion preservatives

Table 3 shows that all formulations were able to inhibit growth of the selected microorganisms and were effective in the Challenge Test.

\section{c) Preliminary stability of emulsions}

i. Heating and cooling: Previous samples were macroscopically examined and analyzed in terms of $\mathrm{pH}$ and stability after centrifugation. This test aims to eliminate unstable formulations to adjust the percentage of constituents. All samples were shown to be stable and kept all initial characteristics except the W/O emulsion IX, where phase separation occurred as described in Table 4. b) Teste da eficácia in vitro dos conservantes presentes nas emulsões

A Tabela 3 mostra que todas as formulações inibiram o crescimento dos microrganismos testados revelando que os conservantes utilizados foram eficazes e cumpriram os critérios estabelecidos pelo Challenge Test.

\section{c) Estabilidade preliminar das emulsões}

i) Aquecimento e arrefecimento: As amostras foram examinadas previamente em termos macroscópicos e analisadas em termos de $\mathrm{pH}$ e estabilidade após centrifugação. Este teste tem como objetivo eliminar formulações instáveis e aferir a percentagem dos constituintes. Como descrito na Tabela 4, todas as amostras demonstraram ser estáveis e mantiveram as características iniciais excepto a emulsão A/O IX onde ocorreu separação das fases. 
Table 3/ Tabela 3. Logarithmic reduction of product along the time Redução logarítmica das UFC/gr de produto ao longo do tempo.

\begin{tabular}{|c|c|c|c|c|c|}
\hline \multicolumn{6}{|c|}{ O/W emulsion XI - Emulsão O/A XI } \\
\hline & & 2 days & 7 days & 14 days & 28 days \\
\hline \multirow{2}{*}{ Bacteria } & $\begin{array}{l}\text { S. aureus } \\
\left(1 \times 10^{6}\right)\end{array}$ & $>3$ & $>3$ & $\begin{array}{c}\text { Increase } \\
\text { Crescimento }\end{array}$ & $\begin{array}{c}\text { Increase } \\
\text { Crescimento }\end{array}$ \\
\hline & $\begin{array}{c}\text { P. aeruginosa } \\
\left(6 \times 10^{6}\right)\end{array}$ & $>3$ & $>3$ & $\begin{array}{c}\text { Increase } \\
\text { Crescimento }\end{array}$ & $\begin{array}{c}\text { Increase } \\
\text { Crescimento }\end{array}$ \\
\hline \multirow{2}{*}{ Fungi } & $\begin{array}{l}\text { C. albicans } \\
\qquad\left(3 \times 10^{5}\right)\end{array}$ & - & - & $>2$ & $\begin{array}{c}\text { Increase } \\
\text { Crescimento }\end{array}$ \\
\hline & $\begin{array}{c}\text { A. brasiliensis } \\
\qquad\left(10 \times 10^{6}\right)\end{array}$ & - & - & $>3$ & $\begin{array}{c}\text { Increase } \\
\text { Crescimento }\end{array}$ \\
\hline \multicolumn{6}{|c|}{ W/O emulsion XIII - Emulsão A/O XIII } \\
\hline \multirow{2}{*}{ Bacteria } & $\begin{array}{l}\text { S. aureus } \\
\left(1 \times 10^{6}\right)\end{array}$ & $>3$ & $>3$ & $\begin{array}{c}\text { Increase } \\
\text { Crescimento }\end{array}$ & $\begin{array}{c}\text { Increase } \\
\text { Crescimento }\end{array}$ \\
\hline & $\begin{array}{c}\text { P. aeruginosa } \\
\left(6 \times 10^{6}\right)\end{array}$ & $>3$ & $>3$ & $\begin{array}{c}\text { Increase } \\
\text { Crescimento }\end{array}$ & $\begin{array}{c}\text { Increase } \\
\text { Crescimento }\end{array}$ \\
\hline \multirow{2}{*}{ Fungi } & $\begin{array}{l}\text { C. albicans } \\
\qquad\left(3 \times 10^{5}\right)\end{array}$ & - & - & $>2$ & $\begin{array}{c}\text { Increase } \\
\text { Crescimento }\end{array}$ \\
\hline & $\begin{array}{l}\text { A. brasiliensis } \\
\qquad\left(10 \times 10^{6}\right)\end{array}$ & - & - & $>3$ & $\begin{array}{c}\text { Increase } \\
\text { Crescimento }\end{array}$ \\
\hline
\end{tabular}


Table 4/ Tabela 4. Results of preliminary test of stability

Resultados do teste preliminar de estabilidade.

\begin{tabular}{|c|c|c|c|c|c|}
\hline \multirow[t]{2}{*}{$\begin{array}{l}\text { Formulations } \\
\text { Formulações }\end{array}$} & \multicolumn{4}{|c|}{$\begin{array}{l}\text { Organoleptic characteristics during heating } \\
\text { and cooling tests } \\
\text { Características organoléticas durante os testes } \\
\text { de arrefeciemto e aquecimento }\end{array}$} & \multirow[t]{2}{*}{$\begin{array}{l}\text { Centrifugation } \\
\text { Centrifugação }\end{array}$} \\
\hline & Before & $48 h$ & $96 h$ & $168 \mathrm{~h}$ & \\
\hline $\begin{array}{l}\text { O/W emulsion } \\
\text { V } \\
\text { Emulsão O/A V }\end{array}$ & $\mathrm{N}$ & $\mathrm{N}$ & $\mathrm{N}$ & $\mathrm{N}$ & $\mathrm{N}$ \\
\hline $\begin{array}{l}\text { O/W emulsion } \\
\text { XI } \\
\text { Emulsão } \\
\text { XI }\end{array}$ & $\mathrm{N}$ & $\mathrm{N}$ & $\mathrm{N}$ & $\mathrm{N}$ & $\mathrm{N}$ \\
\hline $\begin{array}{l}\text { W/O emulsion } \\
\text { IX } \\
\text { Emulsão A/O } \\
\text { IX }\end{array}$ & $\mathrm{N}$ & PS & PS & PS & PS \\
\hline $\begin{array}{l}\text { W/O emulsion } \\
\text { XIII } \\
\text { Emulsão A/O } \\
\text { XIII }\end{array}$ & $\mathrm{N}$ & $\mathrm{N}$ & $\mathrm{N}$ & $\mathrm{N}$ & $\mathrm{N}$ \\
\hline
\end{tabular}

N- normal; PS- phase separation/separação de fases

\section{d) Accelerated stability}

After twelve days, all samples presented the initial organoleptic characteristics. At the 14th day, the $\mathrm{pH}$ and organoleptic properties did not change for the $\mathrm{O} / \mathrm{W}$ emulsion (Table 5). For W/O emulsions, the viscosity did not change (Table 6 ). When the W/O emulsion XIII was exposed to high temperatures, a slight change of color and odour occurred, likely due to the presence of cod liver oil or an insufficient amount of antioxidant. Consequently, the addition of perfume may be needed to mask that unpleasant odour. In the temperature cycles test, the $\mathrm{pH}$ did not change in any sample.

e) Preliminary safety test: No adverse reactions were observed in all tested formulations. d) Estabilidade acelerada das emulsões

Após 12 dias, todas as amostras mantiveram as características organoléticas iniciais. Como descrito na Tabela 5 , no $14^{\circ}$ dia do estudo, o pH não variou para as emulsões $\mathrm{O} / \mathrm{A}$. Para as emulsões A/O, a viscosidade manteve-se constante (Tabela 6). As propriedades organoléticas das emulsões $\mathrm{O} / \mathrm{A}$ permaneceram inalteráveis. Contudo, quando se submeteu a emulsão A/O XIII a temperaturas elevadas, observou-se uma ligeira modificação da cor e do odor, provavelmente devido à presença de óleo de fígado de bacalhau ou a uma quantidade insuficiente de antioxidante. Por conseguinte, e para este caso, poderá ser necessário adicionar um perfume de forma a ocultar o odor desagradável. No caso do teste dos ciclos de temperatura não se observaram variações de $\mathrm{pH}$.

\section{e) Teste preliminar de segurança}

Não foram observadas reacções adversas para todas as formulações testadas. 
Table 5/ Tabela 5. $\mathrm{pH}$ results

Resultados de $\mathrm{pH}$.

\begin{tabular}{|c|c|c|c|c|}
\hline & \multirow[b]{2}{*}{ Days } & $20 \pm 5^{\circ} \mathrm{C}$ & $-5 \pm 2^{\circ} \mathrm{C}$ & $50 \pm 2^{\circ} \mathrm{C}$ \\
\hline & & $\begin{array}{c}\text { Mean } \pm \text { SD } \\
\text { Média } \pm \text { Desv. } \\
\text { Padrão }\end{array}$ & $\begin{array}{c}\text { Mean } \pm \text { SD } \\
\text { Média } \pm \text { Desv. } \\
\text { Padrão }\end{array}$ & $\begin{array}{c}\text { Mean } \pm \text { SD } \\
\text { Média } \pm \text { Desv. } \\
\text { Padrão }\end{array}$ \\
\hline \multirow{3}{*}{$\begin{array}{c}\text { O/W emulsion } \\
\text { V } \\
\text { Emulsão O/A V }\end{array}$} & $3^{\text {th }} / 3^{o}$ & $\mathbf{6 . 8 7} \pm 0.01$ & $\mathbf{6 . 8 9} \pm 0.01$ & $\mathbf{6 . 9 5} \pm 0.03$ \\
\hline & $7^{\text {th }} / 7^{o}$ & $6.89 \pm 0.01$ & $6.89 \pm 0.01$ & $6.94 \pm 0.02$ \\
\hline & $14^{\text {th }} / 14^{o}$ & $6.84 \pm 0.02$ & $6.87 \pm 0.03$ & $6.95 \pm 0.01$ \\
\hline \multirow{3}{*}{$\begin{array}{c}\text { O/W emulsion } \\
\text { XI } \\
\text { Emulsão O/A } \\
\text { XI } \\
\end{array}$} & $3^{\text {th }} / \mathbf{3}^{\mathrm{o}}$ & $6.68 \pm 0.01$ & $\mathbf{6 . 6} \pm 0.02$ & $6.7 \pm 0.01$ \\
\hline & $7^{\text {th }} / 7^{o}$ & $6.68 \pm 0.02$ & $6.62 \pm 0.03$ & $6.71 \pm 0.03$ \\
\hline & $14^{\text {th }} / 14^{o}$ & $6.69 \pm 0.01$ & $\mathbf{6 . 6 3} \pm 0.01$ & $\mathbf{6 . 6 8} \pm 0.00$ \\
\hline
\end{tabular}

Table 6/ Tabela 6. Viscosity results (mean value \pm standard deviation, with torque value)

Resultados de viscosidade (valor médio \pm desvio padrão, com valor de torque).

\begin{tabular}{|c|c|c|c|c|}
\hline \multirow{3}{*}{$\begin{array}{l}\text { Emulsion } \\
\text { Emulsão }\end{array}$} & \multirow{3}{*}{$\begin{array}{l}\text { Days } \\
\text { Dias }\end{array}$} & $20 \pm 5^{\circ} \mathrm{C}$ & $-5 \pm 2^{\circ} \mathrm{C}$ & $50 \pm 2^{\circ} \mathrm{C}$ \\
\hline & & $\operatorname{Mean}\left(\mathrm{X10}^{3}\right) \pm \mathrm{SD}$ & $\operatorname{Mean}\left(\mathrm{X10}^{3}\right) \pm \mathrm{SD}$ & $\operatorname{Mean}\left(X_{10}{ }^{3}\right) \pm S D$ \\
\hline & & $\begin{array}{c}\text { Média }\left(\mathrm{X} 10^{3}\right) \pm \mathrm{SD} \\
\text { (torque) }\end{array}$ & $\begin{array}{c}\text { Média }\left(\mathrm{X10}^{3}\right) \pm \mathrm{SD} \\
\text { (torque) }\end{array}$ & $\begin{array}{c}\text { Média }\left(\mathrm{X}^{3}{ }^{3}\right) \pm \mathrm{SD} \\
\text { (torque) }\end{array}$ \\
\hline \multirow{3}{*}{$\begin{array}{c}\mathbf{O} / \mathrm{W}-\mathbf{V} \\
\mathbf{O} / \mathbf{A}-\mathbf{V}\end{array}$} & $3^{\text {th }} / 3^{o}$ & $72 \pm 50(\% 27.2)$ & $73.6 \pm 13(\% 28.4)$ & $71.5 \pm 17(\% 28)$ \\
\hline & $7^{\text {th }} / 7^{0}$ & $73.5 \pm 65(\% 27.8)$ & $74.0 \pm 40(\% 28)$ & $72.0 \pm 19(\% 28.4)$ \\
\hline & $14^{\text {th }} / 14^{o}$ & $74.0 \pm 42(\% 28)$ & $74.2 \pm 75(\% 27.5)$ & $72.5 \pm 21(\% 28.1)$ \\
\hline \multirow{3}{*}{$\begin{array}{l}\text { O/W-XI } \\
\text { O/A-XI }\end{array}$} & $3^{\text {th }} / 3^{o}$ & $86.4 \pm 50(\% 21.6)$ & $84.8 \pm 34(\% 21.2)$ & $84.8 \pm 10(\% 21.2)$ \\
\hline & $7^{\text {th }} / 7^{0}$ & $84.0 \pm 32(\% 21.0)$ & $84.8 \pm 21(\% 21.2)$ & $84.4 \pm 28(\% 21.1)$ \\
\hline & $14^{\text {th }} / 14^{o}$ & $84.4 \pm 80(\% 21.1)$ & $85.6 \pm 48(\% 21.4)$ & $85.6 \pm 17(\% 21.4)$ \\
\hline \multirow{3}{*}{$\begin{array}{l}\text { W/O-XIII } \\
\text { A/O-XIII }\end{array}$} & $3^{\text {th }} / 3^{o}$ & $81.2 \pm 15(\% 20.3)$ & $81.6 \pm 32(\% 20.4)$ & $81.6 \pm 12(\% 20.4)$ \\
\hline & $7^{\text {th }} / 7^{o}$ & $80.8 \pm 30(\% 20.2)$ & $81.2 \pm 40(\% 20.3)$ & $80.8 \pm 15(\% 20.2)$ \\
\hline & $14^{\mathrm{th}} / 14^{\mathrm{o}}$ & $82.0 \pm 10(\% 20.5)$ & $81.6 \pm 5(\% 20.4)$ & $81.6 \pm 28(\% 20.4)$ \\
\hline
\end{tabular}




\section{Discussion}

The market for natural products has been impressively growing. The "go green" concepts actually may encourage the consumers" urge for "natural", a term traditionally associated to "purity" and "product quality". But today, this term clearly evokes further concepts in the consumer's mind such as "environmentally (eco) friendly", "sustainability" and, of course, "health," and this fact is particularly true in the global cosmetic market. According to the Natural Marketing Institute surveys, approximately $59 \%$ of women prefer cosmetics with $100 \%$ natural ingredients.

Sensory properties are also crucial parameters to be considered in the development of cosmetics. Spreading of a cosmetic during its application on the skin is reportedly a determinant textural attribute whose importance surpasses the product performance ${ }^{(19)}$. All our tested formulations exhibited easy spreadability. However, the type of vehicle and composition does influence the final product aspect.

The cosmetic stability is also a major determinant. In our study, $\mathrm{O} / \mathrm{W}$ emulsion XI and W/O emulsion XIII were the most stable. Final $\mathrm{pH}$ and $\mathrm{pH}$ variation with time or temperature are also predictors of the product's stability. The formulation's (O/W emulsions) $\mathrm{pH}$ did not change during the stability assessment, but it was slightly higher than the normal $\mathrm{pH}$ of the skin. In this case, buffers based on lactic acid and other organic acids might be used during formulation design to control the $\mathrm{pH}$. By adding acid to the aqueous phase before the emulsification step may also help to stabilize the emulsion. However, the amount of acid to be added is critical due to the zinc component. In fact, one of the most important considerations with zinc oxide incorporation in cosmetics formulations is the $\mathrm{pH}$, as it will affect the level of zinc ions in the aqueous phase of the emulsion. Zinc oxide becomes slightly soluble with pHs below 7.5, with a sharp increase below $\mathrm{pH}$ 7. This might cause thickening and/or emulsion instability (7, 14). The choice for these combinatios ofnatural extracts and zinc oxide is also justified mainly due to their reported anti-inflammatory and antiseptic actions and regenerating capacities ${ }^{(4,12)}$. Additionally, zinc oxide also imparts some natural antimicrobial properties and may allow a reduction in the amount of preservatives used ${ }^{(9,13,17}$ we have advanced in our development of a novel diaper that delivers dermatological formulations to help protect the skin from over-hydration and irritation. $\lfloor n \backslash$ nOBJECTIVE: To determine the clinical benefits of a novel disposable diaper designed to deliver a zinc oxide and petrolatum-based formulation continuously to the

\section{Discussão}

$\mathrm{O}$ mercado dos produtos naturais tem vindo a crescer de forma impressionante. O termo "go green" ajuda o consumidor a perceber o aparecimento de uma panóplia de produtos cosméticos contendo produtos naturais, termo tradicionalmente associado à "pureza" e "qualidade de produto". Mas, atualmente, este termo alerta o consumidor para outros tais como "enviromentally (eco) friendly" e, claro, "saúde" e este facto é particularmente verdadeiro no mercado de cosméticos global. De acordo com inquéritos do Natural Marketing Institute cerca $59 \%$ das mulheres preferem cosméticos baseados em ingredientes com origem $100 \%$ natural. As propriedades sensoriais são também parâmetros cruciais a serem considerados no desenvolvimento de cosméticos. A espalhabilidade de creme cosmético é uma propriedade determinante que exclui as performances do produto durante sua aplicação na pele ${ }^{(19)}$. Todas formulações testadas demostraram uma fácil espalhabilidade. Porém, o tipo de veículo e composição influencia o aspeto do produto final.

A estabilidade de um produto cosmético, uma vez aplicado na pele para exercer o efeito desejado é também um fator importante. Neste caso, emulsão O/A XI e a emulsão A/O XIII foram as mais estáveis. Outro parâmetro para prever a estabilidade do produto é, por exemplo, o $\mathrm{pH}$ final do produto e variação de $\mathrm{pH}$ ao longo do tempo ou temperatura. $\mathrm{O}$ pH das formulações (emulsões $\mathrm{O} / \mathrm{A}$ ) não se alterou durante a avaliação da estabilidade mas foi ligeiramente mais elevado do que o pH normal da pele. Neste caso, certos tampões baseados em ácido láctico ou outros ácidos orgânicos são frequentemente utilizados durante a formulação para controlar o pH.. A adição de ácido à fase aquosa antes do passo de emulsificação poderia de facto ajudar a estabilizar a emulsão. Porém, a quantidade de ácido a adicionar é crítica devido ao zinco. De facto, um das mais importantes considerações com formulações com óxido de zinco é o pH da formulação, uma vez que este irá afetar o nível de iões de zinco na fase aquosa de uma emulsão. $\mathrm{O}$ óxido de zinco torna-se ligeiramente solúvel a valores de $\mathrm{pH}$ abaixo de 7,5, com um aumento acentuado abaixo 7. Este facto pode provocar o espessamento e/ ou a instabilidade da emulsão ${ }^{(7,14)}$.

A utilização de extratos naturais e óxido de zinco é justificada principalmente devido às suas ações anti-inflamatórias e antissépticas e de regeneração da pele ${ }^{(4,12)}$. O óxido de zinco tem também algumas propriedades anti-microbianas naturais e pode permitir uma redução da quantidade do agente conservante utilizado ${ }^{(9,13,17}$ we have advanced in our development of a novel diaper that delivers dermatological formulations to help protect the skin from over-hydration and irritation. $\mid n \backslash$ nOBJECTIVE: To determine the clinical benefits of a novel disposable diaper designed to deliver a zinc oxi- 
skin during use. In $\backslash$ nMETHODS: All studies were independent, blinded, randomized clinical trials. Study A was conducted to confirm transfer of the zinc oxide/petrolatum $(\mathrm{ZnO} / \mathrm{Pet})$. As demonstrated by the Challenge Test, extracts or zinc oxide alone were insufficient to ensure inhibition of microorganism growth, therefore,

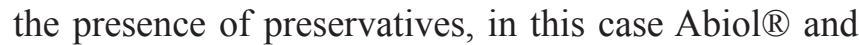
Neolon ${ }^{\circledR}$ 950, was necessary. For the cleansing solution, preservatives were not needed due to the sodium hypochlorite content and its strong antibacterial action. Finally, the hydration effect is extremely dependent on the product composition. Natural extracts may influence the hydration of the skin. Various studies involving aloe vera have demonstrated that this extract has properties that are effective in a variety of skin conditions, from dry skin and burn injuries, to hair and scalp problems $(3,4,20)$. Chamomilla recutita is similarly widely used in cosmetics because it typically reduces redness in the skin ${ }^{(21)}$. This extract contains active flavonoids (also vitamin B), which are particularly useful in sensitive skin care, including dermatitis due to the inhibition of histamine release ${ }^{(11,12)}$.

Formulations developed here appear to present an interesting compromise between naturals and synthetics, as well as cosmetic and skin care effects, and these results justify further studies and development.

\section{Conclusion}

This study describes the initial stage of development and characterization of cosmetics with natural extracts. Products were stable and the results of the safety test suggest that cosmetics were safe. However, they must undergo cosmetic in vivo efficacy testing and additional testing, such as long-term stability and compatibility between the design and the material of the primary packaging.

\section{Conflict of Interest}

The authors declare that there is no financial or personal relationship that can be understood as representing a potential conflict of interest. de and petrolatum-based formulation continuously to the skin during use. $\backslash n \backslash n M E T H O D S$ : All studies were independent, blinded, randomized clinical trials. Study A was conducted to confirm transfer of the zinc oxide/ petrolatum $(\mathrm{ZnO} /$ Pet). No entanto, como se verificou no Challenge Test, por si só, quer os extratos quer o óxido de zinco revelaram-se insuficientes para inibir o crescimento de microrganismos e, por isso, se justifica a presença de dois conservantes: Abiol ${ }^{\circledR}$ e Neolon ${ }^{\circledR} 950$. Na solução de limpeza, o hipoclorito de sódio foi usado devido à sua forte ação antibacteriana e, neste caso, não foi necessário adicionar agentes conservantes.

Finalmente, o nível de hidratação da pele após aplicação de um cosmético é fortemente dependente da composição do produto. Extratos naturais podem influenciar a hidratação da pele. Vários estudos anteriores que incluíram Aloé vera revelaram que este extracto possui propriedades que são eficazes no tratamento de uma variedade de condições alterações dermatológicas da pele escamosa ou seca, doenças cutâneas, problemas no cabelo e couro cabeludo e muito mais ${ }^{(3,4,20)}$. No caso da Chamomilla Recutita, este outro é um extrato muito utilizado em cosméticos porque geralmente reduz a vermelhidão na pele ${ }^{(21)}$. Além disso, o extrato de Chamomilla Recutita contém flavonóides ativos (também Vitamina B), que são particularmente úteis para o cuidado da pele sensível. Estes flavonóides tratam a inflamação relacionada com a dermatite, através da inibição da libertação de histamina.

As formulações aqui desenvolvidas parecem apresentar um compromisso interessante entre produtos naturais e de síntese e de cosméticos bem como efeitos de cuidados da pele, e estes resultados justificam mais estudos e desenvolvimentos.

\section{Conclusão}

Este estudo descreve o desenvolvimento inicial e respectiva caracterização de cosméticos com extratos naturais. Os produtos revelaram-se estáveis e os resultados do teste de segurança sugerem que os cosméticos foram seguros. No entanto, estas formulações serão submetidas a testes de eficácia in vivo e teste adicionais, tais como estabilidade a longo prazo e a compatibilidade entre o design e o material da embalagem primária.

\section{Conflito de interesses}

Os autores declaram não existir qualquer relação de natureza financeira ou pessoal que possa ser entendida ou representar um potencial conflito de interesses. 


\section{References/ Referências}

1. Nicol NH. Anatomy and physiology of the skin. Dermatol Nurs. 2005;17(1):62. doi:10.1016/S0007-1226(78)90109-1.

2. Lambers H, Piessens S, Bloem A, Pronk H, Finkel P. Natural skin surface $\mathrm{pH}$ is on average below 5, which is beneficial for its resident flora. Int J Cosmet Sci. 2006;28(5):359370 .

3. Beikert FC, Schönfeld BS, Frank U, Augustin M. [Antiinflammatory potential of seven plant extracts in the ultraviolet erythema test. A randomized, placebo-controlled study]. Hautarzt. 2013;64(1):40-46

4. Davis RH, Donato JJ, Hartman GM, Haas RC. Anti-inflammatory and wound healing activity of a growth substance in Aloe vera. $\mathrm{J}$ Am Podiatr Med Assoc. 1994;84(2):77-81.

5. Herman A, Herman AP, Domagalska BW, Młynarczyk A. Essential oils and herbal extracts as antimicrobial agents in cosmetic emulsion. Indian J Microbiol. 2013;53(2):232-237.

6. Khan AW, Kotta S, Ansari SH, Sharma RK, Kumar A, Ali J. Formulation development, optimization and evaluation of aloe vera gel for wound healing. Pharmacogn Mag. 2013;9(Suppl 1):S6-S10.

7. Rowe RC, Sheskey PJ, Quinn ME. Handbook of Pharmaceutical Excipients. USA: Pharmaceutical Press; 2009.

8. Terkelsen LH, Eskild-Jensen A, Kjeldsen H, Barker JH, Hjortdal VE. Topical application of cod liver oil ointment accelerates wound healing: an experimental study in wounds in the ears of hairless mice. Scand J Plast Reconstr Surg Hand Surg. 2000;34(1):15-20.
9. Baldwin S, Odio MR, Haines SL, O'Connor RJ, Englehart JS, Lane AT. Skin benefits from continuous topical administration of a zinc oxide/petrolatum formulation by a novel disposable diaper. J Eur Acad Dermatol Venereol. 2001;15 Suppl 1:5-11.

10. Cheng S, Leow YH, Goh CL, Goon A. Contact sensitivity to preservatives in Singapore: frequency of sensitization to 11 common preservatives 2006-2011. Dermat contact, atopic, Occup drug. 25(2):77-82.

11. Srivastava JK, Shankar E, Gupta S. Chamomile: A herbal medicine of the past with bright future. Mol Med Rep. 2010;3(6):895901.

12. Singh O, Khanam Z, Misra N, Srivastava MK. Chamomile (Matricaria chamomilla L.): An overview. Pharmacogn Rev. 2011;5(9):82-95.

13. Hayashi S, Takeshita H, Nagao N, Nikaido $\mathrm{O}$, Miwa N. The relationship between UVB screening and cytoprotection by microcorpuscular $\mathrm{ZnO}$ or ascorbate against DNA photodamage and membrane injuries in keratinocytes by oxidative stress. J Photochem Photobiol B. 2001;64(1):27-35.

14. Heng KY, Kei TY, Singh KJ, Hairui L, AiLing P, Lifeng K. Handbook of Cosmeceutical Excipients and Their Safeties. Elsevier Science; 2014
15. Farmacopeia Portuguesa e Suplementos. Infarmed. 2008. http://www.infarmed.pt/portal/ page/portal/INFARMED/PUBLICACOES/ TEMATICOS/FARMACOPEIA PORTUGUESA_SUPLEMENTOS

16. Frosch P.J. \& Kligmann A.M., The Duhring Chamber: an improved technique for epicutaneous testing of irritant and allergic reactions. Contact Dermatitis. 1979; 5: 73-81.

17. Devos S.A., Van Der Valk P.G. Epicutaneous patch testing. Eur J Dermatol. 2002; 12 (5): 506-511

18. orld Medical Association, 2013. World Medical Association Declaration of Helsinki: ethical principles for medical research involving human subjects. JAMA 310, 2191-2194.

19. Savary G, Grisel M, Picard C. Impact of emollients on the spreading properties of cosmetic products: a combined sensory and instrumental characterization. Colloids Surf B Biointerfaces. 2013;102:371-378.

20. Gupta VK, Malhotra S. Pharmacological attribute of Aloe vera: Revalidation through experimental and clinical studies. Ayu. 2012;33(2):193-196.

21. Wolff HH, Kieser M. Hamamelis in children with skin disorders and skin injuries: results of an observational study. Eur J Pediatr. 2007;166(9):943-948. 